\title{
A Let-7 MicroRNA SNP in the KRAS 3'UTR Is Prognostic in Early-Stage Colorectal Cancer - Letter
}

\author{
Brid M. Ryan, Ana I. Robles, and Curtis C. Harris \\ Laboratory of Human Carcinogenesis, Center for Cancer Research, National Cancer Institute, \\ Bethesda, MD. 20892
}

In their recently published study (1), Smits and colleagues reported an association between rs61764370 and colorectal cancer (CC) survival of early-stage tumors. Similarly, in our study of 678 (441 controls and 237 cases), we found that rs61764370 was also associated

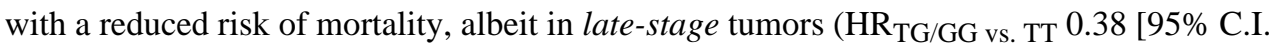
$0.16-0.88] \mathrm{n}=124)$. Neither study found an association with risk.

There are some key differences between the two studies that could explain the differential findings. The first of these relates to the ethnicity of the cohorts. Our study was based upon both African Americans (AA) and European Americans (EA), while that of Smits et al. focused on a European population. While we lose power upon stratification, we still observe the same association in EA and survival of late stage tumors ( $\mathrm{HR}_{\mathrm{TG} / \mathrm{GG}}$ vs. TT 0.46 [95\% C.I. $0.18-1.16] \mathrm{n}=78)$.

We believe a more likely explanation lies in the differences between KRAS mutation status among the two cohorts. In our study, 70\% of the cancers with a KRAS mutation were diagnosed as either stage III or IV. In Smits et al., only 33.5\% of stage III and $43.5 \%$ of stage IV tumors were KRAS mutant. We also noted that there were no deaths among patients who carried rs61764370 and whose tumors were mutant for KRAS. This could therefore suggest that the presence of KRAS mutation is a more important factor in determining the relationship between rs61764370 and survival than stage. In addition, it helps to explain the differences between the studies' findings and clarify why our association was primarily seen in late-stage tumors.

Another possible difference that could confound these reports is the predictive properties of the rs61764370 SNP. Zhang et al. previously reported that the variant $\mathrm{G}$ allele predicts response to single-agent cetuximab monotherapy in refractory $\mathrm{CC}$, particularly in patients whose tumors are KRAS wild-type (2). Intriguingly, Graziano et al. reported that the same genotype was predictive of poor outcome in patients treated with both cetuximab and irinotecan (3). Collectively, these results suggest that both KRAS mutation status, and therapy, modulate the impact of this SNP. Smits et al. did not describe the treatment details of their cohort, and where data are available, our cohort primarily received 5-FU.

Collectively, we find that this SNP is not predictive of risk. Rather, an association with survival has again emerged. However, larger studies that take KRAS, BRAF and treatment status (1-5) into account are needed to more accurately and completely define the role of this SNP in CC and its potential to contribute to individualized and personalized treatment. 


\section{References}

1. Smits KM, Paranjape T, Nallur S, et al. A Let-7 MicroRNA SNP in the KRAS 3'UTR Is Prognostic in Early-Stage Colorectal Cancer. Clin Cancer Res. 2011; 17:7723-31. [PubMed: 21994416]

2. Zhang W, Winder T, Ning Y, et al. A let-7 microRNA-binding site polymorphism in 3'-untranslated region of KRAS gene predicts response in wild-type KRAS patients with metastatic colorectal cancer treated with cetuximab monotherapy. Ann Oncol. 2011; 22:104-9. [PubMed: 20603437]

3. Graziano F, Canestrari E, Loupakis F, et al. Genetic modulation of the Let-7 microRNA binding to KRAS 3'-untranslated region and survival of metastatic colorectal cancer patients treated with salvage cetuximab-irinotecan. Pharmacogenomics J. 2010; 10:458-64. [PubMed: 20177422]

4. Zhang W, Labonte MJ, Lenz HJ. KRAS let-7 LCS6 SNP predicts cetuximab efficacy in KRASwt metastatic colorectal cancer patients: Does treatment combination partner matter? Ann Oncol. 2011; 22:484-5. [PubMed: 21278223]

5. Ruzzo A, Canestrari E, Galluccio N, et al. Role of KRAS let-7 LCS6 SNP in metastatic colorectal cancer patients. Ann Oncol. 2011; 22:234-5. [PubMed: 20926546] 

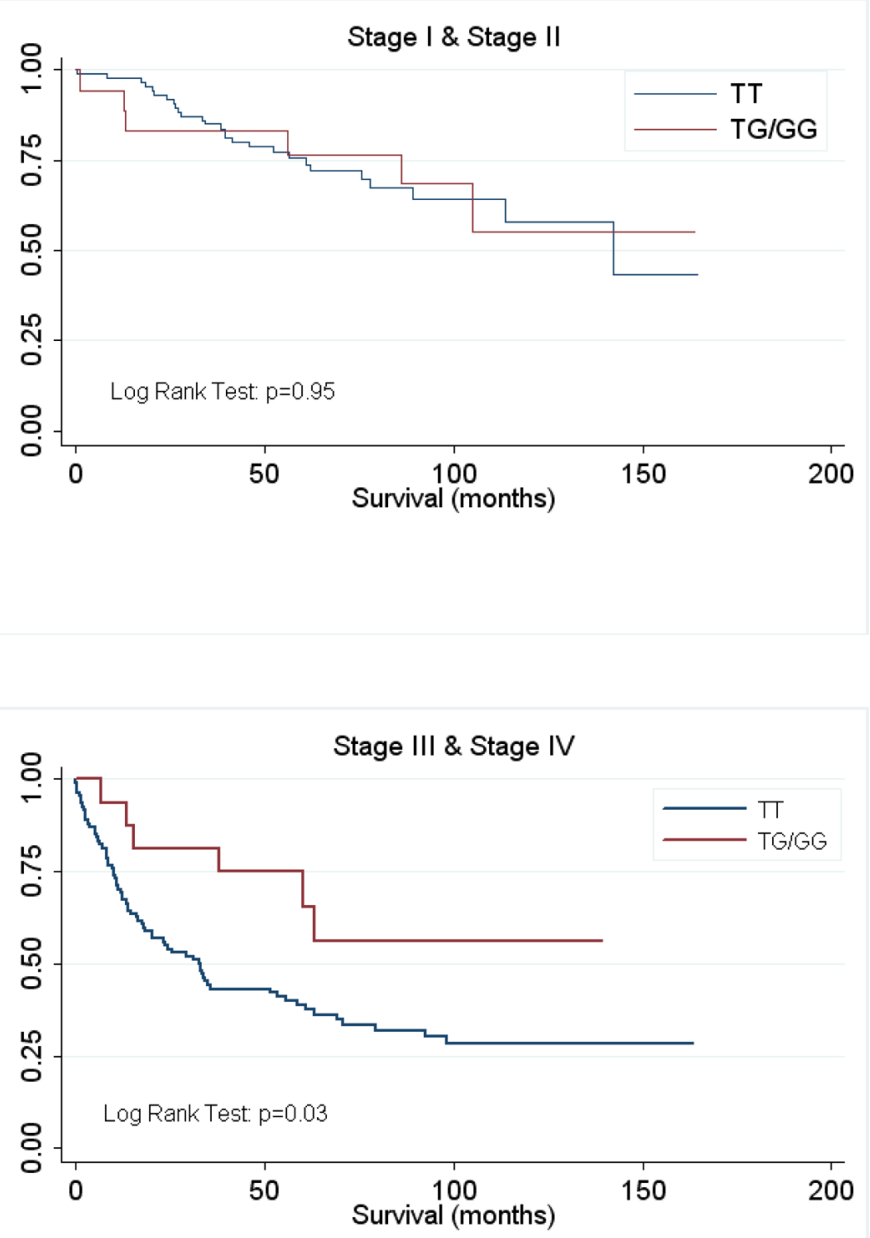

Figure 1.

rs61764370 and survival in colorectal cancer stratified by stage 


\section{Table 2}

Survival of colorectal cancer patients

\begin{tabular}{lllll}
\hline Gene & Genotype & HR & $\mathbf{9 5 \%}$ CI* & p value $^{*}$ \\
\hline Stage I \& II & TT & Reference & & \\
& TG/GG & 0.96 & $0.40-2.36$ & 0.938 \\
Stage III \& IV & TT & $\begin{array}{l}\text { Reference } \\
\end{array}$ & & \\
TG/GG & $\mathbf{0 . 3 8}$ & $\mathbf{0 . 1 7 - 0 . 9 2}$ & $\mathbf{0 . 0 2 5}$ \\
\hline
\end{tabular}

Adjusted for age and gender (Cox regression model) 